\title{
Case-Matched Comparison of the Retroperitoneal Approach With Laparotomy for Necrotizing Pancreatitis
}

\author{
Hjalmar C. van Santvoort • Marc G. Besselink • Thomas L. Bollen · Erik Buskens • \\ Bert van Ramshorst · Hein G. Gooszen · Dutch Acute Pancreatitis Study Group
}

Published online: 16 June 2007

(C) Société Internationale de Chirurgie 2007

\begin{abstract}
Background Minimally invasive necrosectomy through a retroperitoneal approach is gaining popularity for the treatment of necrotizing pancreatitis. There is, however, no substantial evidence from comparative studies in favor of this technique over laparotomy. The aim of this case-matched study was to perform the first head-to-head comparison of necrosectomy by the retroperitoneal approach with laparotomy in patients with necrotizing pancreatitis.

Methods Between 2001 and 2005, there were 15 of 841 consecutive acute pancreatitis patients who underwent necrosectomy by the retroperitoneal approach using a small flank incision. These patients were matched for the presence of preoperative organ failure, status of infection, timing of surgery, age, and computed tomography severity index score
\end{abstract}

H. C. van Santvoort · M. G. Besselink .

H. G. Gooszen $(\varangle)$

Department of Surgery, University Medical Center Utrecht,

Room G.04.228, PO Box 85500, 3508 GA, Utrecht,

The Netherlands

e-mail: h.gooszen@umcutrecht.nl

H. C. van Santvoort

e-mail: h.vansantvoort@umcutrecht.nl

M. G. Besselink · B. van Ramshorst

Department of Surgery, St. Antonius Hospital, PO Box 2500,

3430 EM Nieuwegein, The Netherlands

T. L. Bollen

Department of Radiology, St. Antonius Hospital, PO Box 2500,

3430 EM Nieuwegein, The Netherlands

E. Buskens

Julius Center for Health Sciences and Primary Care, University Medical Center Utrecht, PO Box 85500, 3430 EM Nieuwegein, The Netherlands with 15 of 46 patients treated with necrosectomy by laparotomy and continuous postoperative lavage (CPL).

Methods In addition to all matched preoperative characteristics, there were no significant differences in sex, preoperative intensive care unit (ICU) admission, preoperative ICU stay, preoperative APACHE-II scores, and preoperative multiple organ failure (MOF). Postoperative complications requiring reintervention occurred in six patients in each group $(p=1.000)$. Postoperative new-onset MOF occurred in 10 patients in the laparotomy/CPL group versus 2 patients in the retroperitoneal approach group $(p=0.008)$. Six patients died in the laparotomy/CPL group versus 1 patient in the retroperitoneal approach group $(p=0.080)$.

Conclusions The less postoperative organ failure and the trend toward lower mortality may point to a benefit of the retroperitoneal approach over laparotomy. A randomized controlled design is, however, still required to answer definitively the question of which operative technique is preferably for patients with (infected) necrotizing pancreatitis.

In 1998, Gambiez et al. described the results of necrosectomy for acute pancreatitis through a small left flank incision under visualization using a mediastinoscope [1]. This technique aims at minimizing surgical stress in an already critically ill patient, thereby potentially reducing morbidity and mortality. Since then, several relatively small series (median 15 patients, range 5-46 patients) on similar "minimally invasive" retroperitoneal approaches have been published and have shown promising results [27]. Consequently, these techniques are now the preferred method of intervention in several expert centers.

There is, however, little evidence from comparative studies in favor of these techniques, and selection bias may have been the reason for the favorable outcome of the 
minimally invasive techniques. Only one retrospective study comparing necrosectomy by the retroperitoneal approach versus laparotomy has been performed [6]. This study showed a trend toward decreased mortality after the retroperitoneal approach. A head-to-head comparison (e.g., casematched study or randomized controlled trial) has never been performed. Such a study is warranted before widespread introduction of retroperitoneal minimally invasive techniques, especially as the outcome after necrosectomy by laparotomy has improved greatly in recent years [8-11].

We started using the open abdomen strategy (OAS) at our institution in 1988 for planned relaparotomies without closing the abdomen. Because of high morbidity and mortality, we subsequently switched to laparotomy with continuous postoperative lavage (CPL) in 1995 [12]. In a comparative study, we found that the results of laparotomy and CPL still were not satisfactory [13]. As an alternative, in 2001 necrosectomy by the retroperitoneal approach using a small flank incision was introduced.

The aim of the present study was to report our results using the retroperitoneal approach with minimum risk of confounding and selection bias. To do so, a case-matched comparison with patients undergoing laparotomy and CPL was performed. This pilot study was undertaken in preparation for a nationwide randomized controlled trial [14].

\section{Patients and methods}

\section{Patient identification}

A computer database search for the International Classification of Disease (ICD-9) code for acute pancreatitis was performed in all patients admitted to our two hospitals between April 1, 1995 and April 1, 2005. A total of 841 patients with acute pancreatitis were admitted during this period. An additional search using acute pancreatitis operation codes identified 61 consecutive patients who underwent primary pancreatic necrosectomy in both hospitals during this period. For the entire study period, the choice of surgical strategy was based on the surgeon's preference. Operation records of these patients were reviewed, and patients were grouped according to the type of surgical approach initially selected (intention to treat principle).

From 2001 through 2005, a total of 15 patients underwent necrosectomy through the retroperitoneal approach. During this period and several years before (1995-2005), 46 patients underwent laparotomy and CPL. From 1995 through 2000, a total of 10 patients underwent laparotomy with OAS. The OAS group was excluded from further analysis because the goal of this study was to compare the current techniques (i.e., minimally invasive retroperitoneal approach versus laparotomy and CPL). Of these two groups, all computerized medical reports and patient charts were reviewed by two authors (H.C.v.S. and M.G.H.B.) and the following variables were extracted: date of hospital admission, date of first surgical intervention, bacteriology of peripancreatic and pancreatic necrosis, and preoperative organ failure.

\section{Definitions}

Organ failure was defined as the persistence for at least 48 hours of the following: $\mathrm{PaO}_{2}<60 \mathrm{mmHg}$ despite 4 liters of $\mathrm{O}_{2} / \mathrm{min}$ via nasal tube or the need for mechanical ventilation (pulmonary insufficiency); serum creatinine $>177$ $\mathrm{mmol} / \mathrm{L}$ or need for hemofiltration or hemodialysis (renal failure); systolic blood pressure $<90 \mathrm{mmHg}$ or need for catecholamine support (cardiocirculatory insufficiency); and serum calcium $<1.87 \mathrm{mmol} / \mathrm{L}$ or thrombocyte count of $<100 \times 10^{9} / \mathrm{L}$ (metabolic disorders), adapted from the Atlanta symposium [15]. Multiple organ failure (MOF) was defined as failure of two or more organ systems on the same day, persisting at least 48 hours. Postoperative newonset MOF was defined as failure of two or more organ systems on the same day sometime during the course after the primary necrosectomy, persisting at least 48 hours, but that did not exist prior to that moment in time.

Postoperative complications were defined as follows: bowel perforation: abdominal pain, signs of sepsis, pneumoperitoneum [on either computed tomography (CT) or conventional radiograph] and confirmed during operation; bleeding: postoperative signs of hypovolemic shock, sudden decrease of hemoglobin, active hemorrhage confirmed by angiography or during operation; colonic necrosis: abdominal pain, signs of sepsis, suggestive findings on CT (i.e. pneumatosis intestinalis) and confirmed during operation; gastrointestinal fistula: persistent secretion of fecal material from the postoperative drains or via the drainage canal after removal of all drains and confirmed during operation; pancreatic fistula: persisting secretion of fluid with a high amylase level (> $5000 \mathrm{U} / \mathrm{ml}$ ) for more than 14 days from the postoperative drains or via the drainage canal after removal of all drains.

\section{Computed tomography}

All preoperative contrast-enhanced computed tomography (CECT) scans were retrieved and digitalized. A single radiologist (T.L.B.), who was aware of the clinical condition of the patient and the timing of surgery but not aware of the surgical approach performed, reviewed all CECT scans and determined the CT severity index (CTSI) [16] on the last scan performed before percutaneous drainage (PCD) or surgery. Collections were classified by intraabdominal localization and according to a previously described scoring system [17]. Collections were classified as: 
left (left lateral border of the collection $\leq 5 \mathrm{~cm}$ from the left abdominal wall); intermediate (left lateral border of the collection $>5 \mathrm{~cm}$ from the midline); or central (left lateral border of the collection $<5 \mathrm{~cm}$ from the midline). Accessibility for placement of a percutaneous drain was assessed.

\section{Case matching}

Each of the 15 patients who underwent necrosectomy by the retroperitoneal approach was matched with one patient treated by laparotomy and CPL for all of the following criteria: (1) organ failure at any time prior to primary necrosectomy (yes or no); (2) infection of pancreatic or peripancreatic necrosis as determined by fine-needle aspiration (FNA) and/or intraoperative culture (yes or no); (3) timing of surgery: number of days admitted before primary necrosectomy ( \pm 7 days, at least 15 days after admission); (4) age ( \pm 10 years); and (5) CTSI ( \pm 2 points). These criteria were chosen because it was anticipated that they reflect the most important prognostic factors. Matching for the date (year) of operation to exclude possible confounding due to time effects was not possible because after 2000 the retroperitoneal approach was increasingly used. To minimize bias introduced by using "historical controls," laparotomy/CPL patients were consecutively enrolled in reversed order (i.e., if more than one laparotomy/ CPL patient could be matched with a patient in the retroperitoneal approach group, the patient operated on most recently was selected).

\section{Data collection of matched cases}

The following variables were extracted from the 30 patients' data: sex; etiology of disease; date of preoperative PCD; indication for first surgical intervention; Acute Physiology and Chronic Health Evaluation (APACHE) II score during the 24 hours prior to the primary necrosectomy; maximum perioperative white blood cell (WBC) count; indication for reintervention; type of reintervention; complications leading to reintervention; postoperative new-onset MOF; duration of intensive care (ICU) stay and hospital admission; and date and cause of in-hospital death or date of hospital discharge.

\section{Surgical strategy}

\section{Indication for intervention}

Intervention was deemed necessary in cases of proven (FNA) or suspected infection of pancreatic necrosis and/or peripancreatic necrosis. Infection was suspected when the acute phase of the disease (1-2 weeks) had subsided and there was a sudden onset of spiking fever and an increase in C-reactive protein (CRP) and leukocytes in the presence of heterogeneous fluid collections on CT (with or without gas bubbles). Regardless of infection, an intervention was postponed whenever possible (absence of organ failure or stable organ failure) to the third or fourth week after onset of disease, as it is known that during the acute phase the systemic inflammatory response syndrome (SIRS) with MOF is due to sterile inflammation rather than to infection associated with pancreatic and/or peripancreatic necrosis [18]. Moreover, by postponing intervention, the infected collections demarcate and become encapsulated, thereby theoretically optimizing the conditions for surgical intervention $[19,20]$. FNA was decreasingly used in our institutions because during the acute phase it had no therapeutic consequences (we still tried to postpone intervention in cases of a positive bacterial culture) and during the late phase a patient with signs of infection but a negative FNA would still undergo intervention. Moreover, FNA is known to yield false-negative results [21].

\section{Laparotomy with CPL}

Laparotomy with CPL was first described by Beger et al. [22]. After a bilateral subcostal or median incision, the lesser sac is entered through the gastrocolic omentum. Blunt débridement of all necrotic tissue is performed. Two double-lumen catheters are inserted through separate incisions and positioned in the retroperitoneal space. Opened ligaments are sutured in an attempt to create a closed compartment for local CPL. Planned reintervention is performed only if case packing materials were left behind in the lesser sac to control diffuse bleeding and it was planned to come back within 48 hours to insert drains for CPL. In case of clinical deterioration, an additional laparotomy for further débridement is performed.

\section{Retroperitoneal approach with $C P L$}

We have recently reported on the technical details of this approach [23]. As the first step a $12 \mathrm{~F}$ to $14 \mathrm{~F}$ percutaneous drain is placed in the collection through the left retroperitoneum. The aim is merely to decompress the collection and drain infected fluid, not to remove the solid components of necrosis. If drainage does not lead to clinical improvement (combined normalization of body temperature and decreased WBC count and CRP level) within the next days, the patient is operated on (Fig. 1).

A 5-cm subcostal incision is made in the left flank. Using the percutaneous drain as a guidewire, the retroperitoneum is entered and necrotic material is débrided with long grasping forceps. When débridement can no 


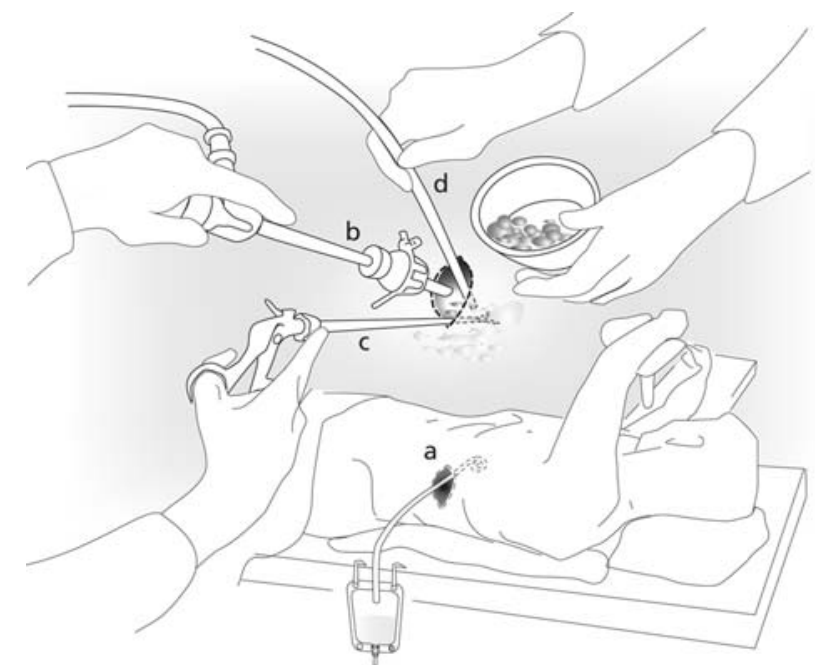

Fig. 1 Retroperitoneal approach in the management of infected necrotizing pancreatitis. Prior to surgical intervention, a computed tomography-guided percutaneous catheter drain is placed in the (peri)pancreatic collection. If there is no clinical improvement, the patient is moved to the operating theater, and the drain is used as a guidewire to open the collection through a small flank incision (a). After the first area of necrosis is removed with a forceps under direct vision, the collection is inspected with a videoscope through a trocar placed in the edge of the incision (b). Additional necrosectomy is performed with a laparoscopic forceps (c) and a suction device (d)

longer be safely performed under direct vision, a 0-degree videoscope is introduced, and the remaining loosely adherent necrotic material is removed. Afterward, two large-bore single-lumen drains are positioned in the cavity exteriorized through the two edges of the incision. The first drain is placed at the deepest possible point of the cavity and the second more superficially. The skin is closed, and CPL is applied with at least 10 liters of normal saline or dialysis fluid per day. Catheters are removed if collapse of the cavity is shown on CT or on a sinogram, and daily production of clear fluids has decreased to less than $50 \mathrm{ml} /$ 24 hours with low amylase and lipase levels. Reintervention is performed only in case of further clinical deterioration.

\section{Statistical analysis}

All statistical analyses were performed using SPSS version 12.01 (SPSS, Chicago, IL, USA). Patients who underwent necrosectomy by the retroperitoneal approach were compared with matched patients treated with laparotomy and CPL, with mortality as the primary outcome measure. The possibility of confounding due to the use of historical controls was assessed by comparing mortality in the laparotomy/CPL group in the first and second half of the study period. Continuous data were shown as the median and range and were compared with the Wilcoxon rank sum test.
For categorical variables, the $\chi^{2}$ test or Fischer's exact test were used as appropriate. A two-tailed $p<0.050$ was considered statistically significant.

\section{Results}

Baseline characteristics

Preoperative patient characteristics are summarized in Table 1 . There were 22 men with a median age of 52 years (34-75 years). Although 14 patients had been referred by other institutions, all primary necrosectomies were performed in our institutions. During the 24 hours preoperatively, 12 patients had organ failure, 8 of whom had failure of two or more organ systems. The median APACHE-II score 24 hours preoperatively was 9 (range 5-20). The median WBC count was $16 \times 10^{9} / \mathrm{L}$ (range $7-33 \times 10^{9} / \mathrm{L}$ ). The median CTSI score was 8 (range 4-10). The median time between admission and primary necrosectomy was 41 days (range 15-164 days). The indication for intervention was proven or suspected infection of pancreatic and/or peripancreatic necrosis in all patients. All patients had heterogeneous collections containing fluid and necrosis on CECT. Eleven patients underwent FNA, which was positive in all cases. Among the patients who underwent PCD, 17 of 18 had positive bacterial cultures. Infection of the pancreatic necrosis was documented by intraoperative culture in 28 patients. There were no patients with a negative culture after FNA or PCD who had a positive culture at a later stage (i.e., intraoperatively).

Adequate matching was achieved for all criteria. Furthermore, there were no differences between the groups for sex, etiology, 24 hours preoperative organ failure (single and multiple), preoperative ICU admission, or 24 hours preoperative WBC count and APACHE-II scores. Preoperative $\mathrm{PCD}$ was performed in six patients in the laparotomy/CPL group. This occurred early in the study period, around the time PCD was introduced in our institution (1997). After this period, PCD was performed only as part of the retroperitoneal approach. Preoperative PCD was not performed in the retroperitoneal approach group in three patients because the collection was adjacent to the left abdominal wall and could be easily reached without a guidewire $(n=2)$ or preoperative ultrasonography was used to locate the retroperitoneal collection $(n=1)$.

\section{Complications requiring reintervention}

In the retroperitoneal approach group there was no need for intraoperative conversion to laparotomy, although four patients required an additional laparotomy during the postoperative course. Postoperative complications requir- 
Table 1 Preoperative characteristics

\begin{tabular}{|c|c|c|c|}
\hline Characteristic & Retroperitoneal approach $(n=15)$ & Laparotomy with CPL $(n=15)$ & $p$ \\
\hline Sex (men) & 12 & 10 & 0.682 \\
\hline Age (years) ${ }^{a}$ & $52(34-66)$ & $53(39-75)$ & 0.325 \\
\hline \multicolumn{4}{|l|}{ Etiology } \\
\hline Biliary & 8 & 5 & 0.462 \\
\hline Alcohol & 3 & 2 & 1.000 \\
\hline Post-ERCP & 1 & 2 & 1.000 \\
\hline Other/ Unknown & 3 & 6 & 0.427 \\
\hline \multicolumn{4}{|l|}{ CT severity index } \\
\hline $4-6$ & 4 & 5 & 1.000 \\
\hline $8-10$ & 11 & 10 & 1.000 \\
\hline \multicolumn{4}{|l|}{ Intraabdominal localization of collection } \\
\hline Left & 9 & 8 & 1.000 \\
\hline Intermediate & 6 & 7 & 1.000 \\
\hline Central & 0 & 0 & 1.000 \\
\hline Accessible for percutaneous drainage & 15 & 15 & 1.000 \\
\hline Preoperative percutaneous catheter drainage & 12 & 6 & 0.060 \\
\hline Organ failure at any time preoperatively & 12 & 12 & 1.000 \\
\hline Organ failure $24 \mathrm{~h}$ preoperatively & 5 & 7 & 1.000 \\
\hline Multiple organ failure $24 \mathrm{~h}$ preoperatively & 4 & 4 & 1.000 \\
\hline Preoperative ICU admission (days) ${ }^{a}$ & $10(1-33)$ & $13(1-44)$ & 0.601 \\
\hline ICU admission $24 \mathrm{~h}$ preoperatively & 5 & 6 & 1.000 \\
\hline APACHE-II score $24 \mathrm{~h}$ preoperatively ${ }^{a}$ & $9(5-18)$ & $9(5-20)$ & 0.902 \\
\hline Time to operation (days) ${ }^{a}$ & $41(15-149)$ & $39(16-164)$ & 0.967 \\
\hline Infected necrosis & 14 & 14 & 1.000 \\
\hline
\end{tabular}

ERCP: endoscopic retrograde cholangiopancreatography; CT: computed tomography; APACHE: Acute Physiology and Chronic Health Evaluation; ICU: intensive care unit

${ }^{a}$ Values are the median and range

ing reintervention are reported in Table 2. There were no significant differences in the incidence of complications in the two groups.

All complications in the laparotomy/CPL group were managed with secondary laparotomy. In the retroperitoneal

Table 2 Postoperative complications requiring reintervention (surgical, endoscopic, radiologic)

\begin{tabular}{llll}
\hline Complication & $\begin{array}{l}\text { Retroperitoneal } \\
\text { approach } \\
(n=15)\end{array}$ & $\begin{array}{l}\text { Laparotomy } \\
\text { with CPL } \\
(n=15)\end{array}$ & $p$ \\
\hline $\begin{array}{l}\text { Patients with one or more } \\
\quad \text { complication }\end{array}$ & 6 & 6 & 1.000 \\
$\begin{array}{l}\text { Total complications } \\
\text { Bowel perforation }\end{array}$ & 8 & 7 & 1.000 \\
Bleeding & 1 & 2 & 1.000 \\
Colonic necrosis & 0 & 1 & 0.330 \\
$\quad \begin{array}{l}\text { Gastrointestinal fistulas } \\
\text { (upper gastrointestinal }\end{array}$ & 1 & 1 & 1.000 \\
$\quad$ tract/colonic & & 3 & 0.598 \\
Pancreatic fistulas & 2 & & 0.483 \\
\hline
\end{tabular}

approach group, complications were managed as follows: (1) bleeding: angiographic coiling $(n=2)$, open packing through the primary left flank incision $(n=1)$, and open packing through a laparotomy $(n=1)$; (2) pancreatic fistulas: endoscopic stent placement in the pancreatic duct ( $n$ $=2)$; (3) bowel perforation: laparotomy $(n=1)$; and (4) gastrointestinal fistula: laparotomy $(n=1)$.

Of the four cases of bleeding that occurred in the retroperitoneal approach group, two occurred after an additional laparotomy was performed for a gastrointestinal fistula $(n=1)$ and after additional necrosectomy $(n=1)$.

\section{Outcome}

Postoperative outcomes are shown in Table 3. Additional necrosectomy was performed according to the treatment strategy originally applied, with the exception of one patient in the retroperitoneal group in whom further débridement by this approach was not deemed feasible. There were no differences in the number of necrosectomies, total number of surgical interventions, postoperative ICU 
Table 3 Postoperative outcomes

${ }^{a}$ Values are the median (range)

\begin{tabular}{llll}
\hline Outcome & $\begin{array}{l}\text { Retroperitoneal } \\
\text { approach }(n=15)\end{array}$ & $\begin{array}{l}\text { Laparotomy with } \\
\text { CPL }(n=15)\end{array}$ & $p$ \\
\hline Surgical reintervention & 12 & 13 & 1.000 \\
$\quad$ For postoperative complication & 3 & 6 & 0.427 \\
$\quad$ For further necrosectomy & 11 & 13 & 0.651 \\
Necrosectomies (total no.) $^{a}$ & $2(1-9)$ & $2(1-13)$ & 0.624 \\
Surgical interventions (total no.) $^{a}$ & $3(1-11)$ & $4(1-14)$ & 0.345 \\
Postoperative new-onset multiple organ failure & 2 & 10 & 0.008 \\
Postoperative ICU admissions $^{\text {Postoperative ICU admissions, survivors (days) }}{ }^{a}$ & 11 & 12 & 1.000 \\
Postoperative hospital stay, survivors (days) $^{a}$ & $9(0-83)$ & $19(0-44)$ & 0.643 \\
Total hospital stays survivors (days) $^{a}$ & $100(45-240)$ & $54(20-150)$ & 0.926 \\
In-hospital mortality & 1 & 6 & 0.600 \\
\hline
\end{tabular}

admissions, or postoperative and total hospital stays between the two groups.

Although the preoperative WBC counts were similar, the median 24-hour postoperative WBC count was lower in the retroperitoneal approach group (11 vs. $18 \times 10^{9} / \mathrm{L}, p=$ 0.02). Postoperative new-onset organ failure occurred in 10 patients in the laparotomy/CPL group versus 2 patients in the retroperitoneal approach group $(p=0.008)$.

Of the 30 patients, 7 died. In the laparotomy/CPL group, six patients died, all because of MOF. These patients had a median APACHE-II score 24 hours preoperatively of 9 (range 6-20), and two of the six patients were admitted to the ICU at the time of surgery. One patient in the retroperitoneal approach group died. The cause of death was a pulmonary embolus 5 weeks after the primary necrosectomy. After a mean postoperative hospital stay of 55 days (range 18-162 days) the surviving 23 patients were discharged from hospital in good clinical condition.

Four of six patients died during the first half of the study (1995-2000) versus two of nine during the second half of the study (2000-2005) $(p=0.136)$.

\section{Discussion}

This study is the first case-matched study comparing necrosectomy by the retroperitoneal approach with laparotomy/ CPL for necrotizing pancreatitis. The main findings are that (1) postoperative new-onset MOF occurred less frequently after the retroperitoneal approach and (2) there was a trend toward less mortality in the retroperitoneal approach group.

Disease severity and the overall mortality rate in this study are in the same range as presented in reports by some expert centers [6, 24-26]. The timing of the intervention (median 41 days) and percentage of infection at the primary intervention (93\%) are among the upper end of data reported in the literature [27].
Outcomes after the retroperitoneal approach compare favorably with other reports, which have cited 53\% morbidity and $18 \%$ mortality rates [1-7]. The suggestion that the retroperitoneal approach is associated with increased complication rates [1-3] was not confirmed by our results. The incidence of complications following the retroperitoneal approach did not differ from that in the laparotomy/ CPL group and was similar to those previously reported after laparotomy [10, 12, 24, 25, 28]. Another suggested disadvantage of the retroperitoneal approach is the need for repeated procedures, resulting in a significantly longer postoperative hospital stay compared to that after necrosectomy by laparotomy $[1-3,6]$. In the current study, however, the number of reinterventions did not differ between groups. This may be explained by the fact that the technique applied in the present study is essentially a semiopen approach. The small incision allows removal of large pieces of necrotic tissues - far larger than is possible with a purely endoscopic approach [2, 5].

How can one explain the trend toward improved outcome after the retroperitoneal approach? A possible explanation is that the retroperitoneal approach induces less perioperative and postoperative stress than laparotomy because a small $(5 \mathrm{~cm})$ incision is used, the peritoneum is left intact, and the peritoneal cavity is not contaminated. Several other authors hypothesized that by minimizing the inflammatory "hit'" of necrosectomy the retroperitoneal approach may lessen the risk of postoperative MOF in the already critically ill patient [1-3]. Notably, in the present study, the retroperitoneal approach was associated with significantly less postoperative new-onset organ failure. Moreover, in the laparotomy/CPL group, MOF was the only cause of death. The suggestion of reduced surgical stress is also underlined by the significantly lower postoperative WBC count after the retroperitoneal approach, whereas preoperative values were similar for the two groups. The study by Connor et al. also supports the 
hypothesis of reduced surgical stress using the retroperitoneal approach [6]. In 53\% (47/88) of patients, minimally invasive retroperitoneal necrosectomy was performed with $19 \%$ mortality compared to $39 \%$ mortality after laparotomy $(p=0.06)$. Although no differences in postoperative complication rates were observed, the postoperative APACHE-II score was lower and the postoperative ICU stay shorter in their retroperitoneal group.

In the current study, the risk of selection bias was minimized by matching patients for essentially all criteria known to affect outcome: organ failure [29-32], infection of necrosis [19, 33, 34], timing of intervention [18, 19, 27, 35], age [32, 36-38], and CTSI score [19, 33, 39-41]. We acknowledge that after introduction of the retroperitoneal approach laparotomy/CPL was still performed in some patients. One might therefore argue that there were specific reasons for this (e.g., more extensive necrosis, less accessible collections) and that selection bias was thereby introduced. However, as this was a case-matched design, a control patient was selected from a larger group of patients undergoing laparotomy only if his or her criteria matched those of a patient undergoing the retroperitoneal approach. This meant that only 9 of 25 patients undergoing laparotomy/CPL after introduction of the retroperitoneal approach were included as control patients. As a result of this process, patients were comparable for all of the 15 baseline characteristics, including accessibility and intraabdominal distribution of the peripancreatic collections. The fact that laparotomy/CPL was still performed during the second period of the study is primarily explained by the preference of the designated surgeon at that time. The retroperitoneal approach simply took some time to gain popularity.

Although the sample size is small, the number of patients in this study is at the median of numbers reported in the literature. Nevertheless, the small sample size might have led to a type II statistical error for certain endpoints (e.g., total complications). The statistical power could have been increased by matching cases to controls in a ratio of $1: 2$. It was chosen not to do so because this would have meant making concessions on the matching criteria, resulting in less-comparable groups. In the present study, comparability was preferred over power.

Being left with historical controls for comparative studies is not uncommon when new surgical techniques are enthusiastically implemented in clinical practice [42]. This points out the need for randomized controlled trials performed in a timely fashion (i.e., before an experimental technique has become "routine care" without evidence from well designed comparative studies being available).

Mortality for laparotomy with CPL did not significantly improve during the second half of this study. However, an improvement in supportive treatment (e.g., better intensive care facilities) is likely to have occurred over time. Recent studies show improved outcome after laparotomy, with excellent mortality rates (as low as 6\%) [8-11]. In addition to better supportive care, a possible explanation for this improved outcome is the fact that surgical intervention is increasingly being postponed [18-20, 27].

Although this study represents the highest level of evidence on the subject thus far, the sample size was too small to draw definitive conclusions. Moreover, comparison in a randomized design is still warranted, especially when considering the improvement in outcome after laparotomy in the recent literature. To address this issue, we have recently started a randomized controlled multicenter trial comparing necrosectomy by laparotomy with CPL with a minimally invasive "step-up approach" [14]. Patients are currently being enrolled from 20 hospitals of the Dutch Acute Pancreatitis Study Group.

\section{References}

1. Gambiez LP, Denimal FA, Porte HL, et al. (1998) Retroperitoneal approach and endoscopic management of peripancreatic necrosis collections. Arch Surg 133:66-72

2. Carter CR, McKay CJ, Imrie CW (2000) Percutaneous necrosectomy and sinus tract endoscopy in the management of infected pancreatic necrosis: an initial experience. Ann Surg 232:175-180

3. Horvath KD, Kao LS, Ali A, et al. (2001) Laparoscopic assisted percutaneous drainage of infected pancreatic necrosis. Surg Endosc 15:677-682

4. Castellanos G, Serrano A, Piñero A, et al. (2001) Retroperitoneoscopy in the management of drained infected pancreatic necrosis. Gastrointest Endosc 53:514-515

5. Connor S, Ghaneh P, Raraty M, et al. (2003) Minimally invasive retroperitoneal pancreatic necrosectomy. Dig Surg 20:270-277

6. Connor S, Alexakis N, Raraty MG, et al. (2005) Early and late complications after pancreatic necrosectomy. Surgery 37:499505

7. Castellanos G, Pinero A, Serrano A, et al. (2005) Translumbar retroperitoneal endoscopy: an alternative in the follow-up and management of drained infected pancreatic necrosis. Arch Surg 140:952-955

8. Ashley SW, Perez A, Pierce EA, et al. (2001) Necrotizing pancreatitis: contemporary analysis of 99 consecutive cases. Ann Surg 234:572-579

9. Farkas G, Marton J, Mandi Y, et al. (2006) Surgical management and complex treatment of infected pancreatic necrosis: 18-year experience at a single center. J Gastrointest Surg 10:278-285

10. Fernandez-del Castillo C, Rattner DW, Makary MA, et al. (1998) Debridement and closed packing for the treatment of necrotizing pancreatitis. Ann Surg 228:676-684

11. Hilal MA, Zidan B, Howse F, et al. (2006) Treatment of infected pancreatic necrosis by complete debridement, and closed conventional drainage. Pancreatology 6:398

12. Bosscha K, Hulstaert PF, Hennipman A, et al. (1998) Fulminant acute pancreatitis and infected necrosis: results of open management of the abdomen and "planned" reoperations. J Am Coll Surg 187:255-262

13. Nieuwenhuijs VB, Besselink MG, van Minnen LP, et al. (2003) Surgical management of acute necrotizing pancreatitis: a 13-year experience and a systematic review. Scand J Gastroenterol Suppl (239):111-116 
14. Besselink MG, van Santvoort HC, Nieuwenhuijs VB, et al. (2006) Minimally invasive 'step-up approach' versus maximal necrosectomy in patients with acute necrotizing pancreatitis (PANTER trial): design and rationale of a randomised controlled multicenter trial. BMC Surg 6:6

15. Bradley EL III (1993) A clinically based classification system for acute pancreatitis: summary of the International Symposium on Acute Pancreatitis, Atlanta, Ga, September 11-13, 1992. Arch Surg 128:586-590

16. Balthazar EJ, Robinson DL, Megibow AJ, et al. (1990) Acute pancreatitis: value of $\mathrm{CT}$ in establishing prognosis. Radiology 174:331-336

17. Besselink MG, van Santvoort HC, Schaapherder AF, et al. (2007) Feasibility of minimally invasive approaches in patients with infected necrotizing pancreatitis. Br J Surg 94:604-608

18. Werner J, Feuerbach S, Uhl W, et al. (2005) Management of acute pancreatitis: from surgery to interventional intensive care. Gut 54:426-436

19. Uhl W, Warshaw A, Imrie C, et al. (2002) IAP guidelines for the surgical management of acute pancreatitis. Pancreatology 2002;2:565-573

20. Connor S, Raraty MG, Neoptolemos JP, et al. (2006) Does infected pancreatic necrosis require immediate or emergency debridement? Pancreas 33:128-134

21. Rau B, Pralle U, Mayer JM, et al. (1998) Role of ultrasonographically guided fine-needle aspiration cytology in the diagnosis of infected pancreatic necrosis. Br J Surg 85:179184

22. Beger HG, Buchler M, Bittner R, et al. (1988) Necrosectomy and postoperative local lavage in patients with necrotizing pancreatitis: results of a prospective clinical trial. World J Surg 12:255262

23. Van Santvoort HC, Besselink MG, Horvath KD, et al. (2007) Videoscopic assisted retroperitoneal debridement in infected necrotizing pancreatitis HPB 9:156-159

24. Tsiotos GG, Luque-de Leon E, Sarr MG (1998) Long-term outcome of necrotizing pancreatitis treated by necrosectomy. Br J Surg 85:1650-1653

25. Buchler MW, Gloor B, Muller CA, et al. (2000) Acute necrotizing pancreatitis: treatment strategy according to the status of infection. Ann Surg 232:619-626

26. Rau B, Bothe A, Beger HG (2005) Surgical treatment of necrotizing pancreatitis by necrosectomy and closed lavage: changing patient characteristics and outcome in a 19-year, single-center series. Surgery 138:28-39
27. Besselink MG, Schoenmaeckers EJ, Buskens E, et al. (2007, in press) Timing of surgical intervention in necrotizing pancreatitis. Arch Surg

28. Branum G, Galloway J, Hirchowitz W, et al. (1998) Pancreatic necrosis: results of necrosectomy, packing, and ultimate closure over drains. Ann Surg 227:870-877

29. Tenner S, Sica G, Hughes M, et al. (1997) Relationship of necrosis to organ failure in severe acute pancreatitis. Gastroenterology 113:899-903

30. Halonen KI, Pettila V, Leppaniemi AK, et al. (2002) Multiple organ dysfunction associated with severe acute pancreatitis. Crit Care Med 30:1274-1279

31. Buter A, Imrie CW, Carter CR, et al. (2002) Dynamic nature of early organ dysfunction determines outcome in acute pancreatitis. Br J Surg 89:298-302

32. Banks PA, Freeman ML (2006) Practice guidelines in acute pancreatitis. Am J Gastroenterol 101:2379-2400

33. Anonymous (2005) UK guidelines for the management of acute pancreatitis. Gut 54(Suppl 3):iii1-iii9

34. Besselink MG, de Bruijn MT, Rutten JP, et al. (2006) Surgical intervention in patients with necrotizing pancreatitis. Br J Surg 93:593-599

35. Mier J, Luque-de León E, Castillo A, et al. (1997) Early versus late necrosectomy in severe necrotizing pancreatitis. Am J Surg 173:71-75

36. Connor S, Ghaneh P, Raraty M, et al. (2003) Increasing age and APACHE II scores are the main determinants of outcome from pancreatic necrosectomy. Br J Surg 90:1542-1548

37. Halonen KI, Leppaniemi AK, Puolakkainen PA, et al. (2000) Severe acute pancreatitis: prognostic factors in 270 consecutive patients. Pancreas 21:266-271

38. Company L, Saez J, Martinez J, et al. (2003) Factors predicting mortality in severe acute pancreatitis. Pancreatology 3:144-148

39. Balthazar EJ, Freeny PC, vanSonnenberg E (1994) Imaging and intervention in acute pancreatitis. Radiology 193:297-306

40. van den Biezenbos AR, Kruyt PM, Bosscha K, et al. (1998) Added value of CT criteria compared to the clinical SAP score in patients with acute pancreatitis. Abdom Imaging 23:622-626

41. Simchuk EJ, Traverso LW, Nukui Y, et al. (2000) Computed tomography severity index is a predictor of outcomes for severe pancreatitis. Am J Surg 179:352-355

42. Keus F, de Jong JAF, Gooszen HG, et al. (2006) Laparoscopic versus open cholecystectomy for patients with symptomatic cholecystolithiasis. Cochrane Database of Systematic Reviews, Issue 4. Art. No. CD006231. DOI 10.1002/14651858. CD006231 